\title{
Uncertainty of Oscilloscope Timebase Distortion Estimate
}

\author{
C. M. Wang, Paul D. Hale, Kevin J. Coakley, and Tracy S. Clement
}

\begin{abstract}
We study several problems related to the characterization of the timebase in high-speed sampling oscilloscopes. First, we examine the bias of using the method of the first-order approximation to estimate the additive and time jitter noises, and present a procedure to adjust for the bias in the estimates. We then study the bias and variance of a least-squares timebase distortion estimate that uses multiple sets of waveforms. Based on simulations, a method for calculating the uncertainty of the timebase distortion estimate is proposed. We also study the effects of amplitude and phase drifts, as well as jitter error on the estimation of timebase distortion. Results are shown using simulations with parameters that are closely related to those we observe in our laboratory.
\end{abstract}

Index Terms-Curve fitting, harmonic distortion, least squares methods, mean square error methods, timing jitter.

\section{INTRODUCTION}

$\mathbf{H}$ IGH speed sampling oscilloscopes suffer from several nonideal properties that must be characterized and compensated for. One of these effects is timing errors. At the $i$ th sample, the timing error is the sum of a deterministic timebase distortion (TBD) $h_{i}$, and a random timing jitter error $\tau_{i}$. Thus, the $i$ th sample of the signal of interest $y_{i}$ as a function of time is given by

$$
y_{i}=g\left((i-1) T_{s}+h_{i}+\tau_{i}\right)+\epsilon_{i}
$$

where $T_{s}$ is the target time interval between samples and $\epsilon_{i}$ is additive noise. The jitter and additive noises are independent zero-mean random variables with variances $\sigma_{\tau}^{2}$ and $\sigma_{\epsilon}^{2}$.

The problem of estimating these effects based on sine-wave data has been studied by several authors. Pintelon and Schoukens [1] present a sine-wave fitting procedure for estimating the harmonic distortion of the sampling oscilloscopes assuming there is no TBD. Schoukens, Pintelon, and Vandersteen [2] propose a fitting procedure that eliminates the TBD restriction. Their approach, however, uses a parametric TBD estimation method [3] that is known to perform poorly when the TBD has discontinuities [4]. Recent work [5]-[7] has overcome this problem by estimating a nonparametric TBD based on waveforms of multiple phases and frequencies.

All the above fitting procedures use the weighted nonlinear least squares (LS) for parameter estimation. The weighting

Manuscript received August 18, 2000; revised November 14, 2001. P. Hale was supported in part by the Office of Naval Research and the Space and Naval Warfare Systems Center.

C. M. Wang and K. J. Coakley are with the Statistical Engineering Division, National Institute of Standards and Technology, Boulder, CO 80303 USA.

P. D. Hale and T. S. Clement are with the Optoelectronics Division, National Institute of Standards and Technology, Boulder, CO 80303 USA.

Publisher Item Identifier S 0018-9456(02)01388-8. scheme is to weight each data point proportionally to the inverse of its variance. The variance can be obtained either from independent, repeated measurements or (if we have prior information on the additive and jitter noise variances) from the approximate model

$$
\operatorname{var}\left(y_{i}\right) \approx \sigma_{\epsilon}^{2}+\left(g^{\prime}\left(t_{i}\right)\right)^{2} \sigma_{\tau}^{2}
$$

where $g^{\prime}\left(t_{i}\right)$ is the derivative of the measured signal evaluated at $t_{i}=(i-1) T_{s}+h_{i}$. On the other hand, (2) can be used to estimate $\sigma_{\epsilon}$ and $\sigma_{\tau}$ if repeated measurements and TBD estimate are available. Since (2) is derived from a first-order Taylor expansion of (1) on $\tau$, it is of interest to study the bias of estimating $\sigma_{\epsilon}$ and $\sigma_{\tau}$ using (2). In this paper, we derive the exact formula relating $\operatorname{var}\left(y_{i}\right)$ to $\sigma_{\epsilon}$ and $\sigma_{\tau}$ for the sine-wave data. The result can be used to produce $\sigma_{\epsilon}$ and $\sigma_{\tau}$ estimates which have smaller bias than that of estimates obtained from (2).

In practice, the characterization process starts by selecting a pair of appropriate frequencies to generate waveforms. At each of two frequencies, signals are sampled at different starting phases. It is shown [7] that if multiple sets of waveforms are used to estimate the TBD by averaging, only two signals in quadrature from each frequency are sufficient. In our approach, we measure 100 sets of four waveforms (see [7] for details regarding the estimation of TBD). Although the starting phase for each waveform need not be the same among the 100 sets of measurements, they are often very close and the 100 waveforms are usually treated as repeated measurements. The sample variances are computed from the waveforms and are used to construct the weights in the LS procedures. In this paper, we study the effects of varying phases and amplitudes, as well as varying jitter noises, on TBD estimation, if they were (wrongly) assumed to be constant among waveforms.

The use of 100 sets of four waveforms allows us to examine the variation among the 100 individual TBD estimates. In this paper, we study the bias and variance of the final TBD estimate (the average of the 100 TBD estimates), and propose a method for computing the uncertainty of the final TBD estimate.

\section{Estimation OF AdDitive AND JitTER NoiseS}

We first derive the exact relationship between $\operatorname{var}\left(y_{i}\right)$ and $\sigma_{\epsilon}$ and $\sigma_{\tau}$ for the simplest case

$$
y_{i}=\alpha+\beta \sin \left(2 \pi f\left((i-1) T_{s}+h_{i}+\tau_{i}\right)+\phi\right)+\epsilon_{i}
$$

where $\alpha, \beta, f$, and $\phi$ are, respectively, the offset, amplitude, frequency, and phase of the sine wave. Denote $t_{i}=(i-1) T_{s}+$ 
TABLE I

BIAS FACTORS OF THE FIRST-ORDER APPROXIMATION

\begin{tabular}{cc|cc}
\hline$f / f_{s}$ & $\sigma_{\tau}\left(\%\right.$ of $\left.T_{s}\right)$ & $\sqrt{c_{\tau}}$ & $\sqrt{c_{\epsilon}}$ \\
\hline $1 / 10$ & 25 & 0.9817 & $0.0172 \beta$ \\
$1 / 10$ & 50 & 0.9288 & $0.0665 \beta$ \\
$1 / 10$ & 100 & 0.7461 & $0.2306 \beta$ \\
$1 / 10$ & 150 & 0.5221 & $0.4162 \beta$ \\
$1 / 32$ & 25 & 0.9982 & $0.0017 \beta$ \\
$1 / 32$ & 50 & 0.9928 & $0.0068 \beta$ \\
$1 / 32$ & 100 & 0.9715 & $0.0267 \beta$ \\
$1 / 32$ & 150 & 0.9372 & $0.0588 \beta$ \\
$1 / 100$ & 25 & 0.9998 & $0.0002 \beta$ \\
$1 / 100$ & 50 & 0.9993 & $0.0007 \beta$ \\
$1 / 100$ & 100 & 0.9970 & $0.0028 \beta$ \\
$1 / 100$ & 150 & 0.9934 & $0.0063 \beta$ \\
\hline \multicolumn{4}{r}{}
\end{tabular}

$h_{i}$, and assume that $\epsilon_{i}$ and $\tau_{i}$ are independent Gaussian noises; it is shown in the Appendix that

$$
\begin{aligned}
\operatorname{var}\left(y_{i}\right)= & \beta^{2}\left(1+e^{-8 \pi^{2} f^{2} \sigma_{\tau}^{2}}-2 e^{-4 \pi^{2} f^{2} \sigma_{\tau}^{2}}\right) / 2+\sigma_{\epsilon}^{2} \\
& +\left(g^{\prime}\left(t_{i}\right)\right)^{2}\left(e^{-4 \pi^{2} f^{2} \sigma_{\tau}^{2}}-e^{-8 \pi^{2} f^{2} \sigma_{\tau}^{2}}\right) / 4 \pi^{2} f^{2} .
\end{aligned}
$$

Eq. (4) differs from (2) by a scaling coefficient $\left[\right.$ on $\left(g^{\prime}\left(t_{i}\right)\right)^{2}$ term]

$$
c_{\tau}=\left(e^{-4 \pi^{2} f^{2} \sigma_{\tau}^{2}}-e^{-8 \pi^{2} f^{2} \sigma_{\tau}^{2}}\right) / 4 \pi^{2} f^{2} \sigma_{\tau}^{2}
$$

and an offset

$$
c_{\epsilon}=\beta^{2}\left(1+e^{-8 \pi^{2} f^{2} \sigma_{\tau}^{2}}-2 e^{-4 \pi^{2} f^{2} \sigma_{\tau}^{2}}\right) / 2 .
$$

If we express $\sigma_{\tau}$ in units of the sample period $T_{s}$, then the magnitude of $c_{\tau}$ and $c_{\epsilon}$ depends only on the ratio $f / f_{s}$, where $f_{s}$ is the sampling frequency. Table I displays the square root of $c_{\tau}$ and $c_{\epsilon}$ for some combinations of $\sigma_{\tau}$ and $f / f_{s}$.

Table I indicates that, given the frequency $f$, the poor choice of the sampling frequency $f_{s}$ or large $\sigma_{\tau}$ can cause the firstorder approximation to produce $\sigma_{\tau}$ and $\sigma_{\epsilon}$ estimates with substantial bias. To illustrate this further, simulations were conducted. Repeated waveforms were generated from a nominal sine wave with an amplitude of $0.2 \mathrm{~V}$ and a frequency of 16 $\mathrm{GHz}$. The measurements were sampled at 2048 points in a time window of $4 \mathrm{~ns}$. This yields $f / f_{s}=1 / 32$. One value of $\sigma_{\epsilon}$, $0.002 \mathrm{~V}$ ( $1 \%$ of the amplitude), and two values of $\sigma_{\tau}, 1 \mathrm{ps}$ (about $50 \%$ of the sample period) and $2 \mathrm{ps}$, were used. Based on the generated waveforms, the method of the first-order approximation was employed to estimate $\sigma_{\epsilon}$ and $\sigma_{\tau}$. The process was repeated 100 times. Table II displays the means of the $100 \sigma_{\epsilon}$ and $\sigma_{\tau}$ estimates for sample sizes (number of repeated waveforms) 150 and 300.

The (empirically obtained) bias of the $\sigma_{\epsilon}$ and $\sigma_{\tau}$ estimates in Table II agrees well with the (analytical) results in Table I. It shows that the first-order approximation can adequately estimate the jitter noise variance but not the additive noise variance for the sampling frequency considered.

To reduce the estimation bias, one can work directly on (4) to obtain the parameter estimates using the nonlinear LS. A simpler method is to adjust the results of the first-order approximation based on (4). Specifically, let $\tilde{\sigma}_{\epsilon}$ and $\tilde{\sigma}_{\tau}$ be the estimates of
TABLE II

ADDITIVE NOISE AND JITTER ESTIMATES USING THE FIRST-ORDER APPROXIMATION

\begin{tabular}{c|cc|cc}
\hline \multirow{2}{*}{ sample size } & \multicolumn{2}{|c|}{ nominal } & \multicolumn{2}{c}{ estimated } \\
\cline { 2 - 5 } & $\sigma_{\epsilon}(\mathrm{V})$ & $\sigma_{\tau}(\mathrm{ps})$ & $\sigma_{\epsilon}(\mathrm{V})$ & $\sigma_{\tau}(\mathrm{ps})$ \\
\hline 150 & 0.002 & 1 & 0.0025 & 0.99 \\
150 & 0.002 & 2 & 0.0060 & 1.94 \\
300 & 0.002 & 1 & 0.0025 & 0.99 \\
300 & 0.002 & 2 & 0.0060 & 1.94 \\
\hline
\end{tabular}

TABLE III

AdDitive NoISE AND JitTer Estimates Using THE ADJUSTED FIRST-ORDER APPROXIMATION

\begin{tabular}{c|cc|cc}
\hline \multirow{2}{*}{ sample size } & \multicolumn{2}{|c|}{ nominal } & \multicolumn{2}{c}{ estimated } \\
\cline { 2 - 5 } & $\sigma_{\epsilon}(\mathrm{V})$ & $\sigma_{\tau}(\mathrm{ps})$ & $\sigma_{\epsilon}(\mathrm{V})$ & $\sigma_{\tau}(\mathrm{ps})$ \\
\hline 150 & 0.002 & 1 & 0.0020 & 1.00 \\
150 & 0.002 & 2 & 0.0019 & 2.00 \\
300 & 0.002 & 1 & 0.0020 & 1.00 \\
300 & 0.002 & 2 & 0.0020 & 2.00 \\
\hline
\end{tabular}

$\sigma_{\epsilon}$ and $\sigma_{\tau}$ obtained using the first-order approximation, then the bias-adjusted estimates, denoted by $\hat{\sigma}_{\epsilon}$ and $\hat{\sigma}_{\tau}$, are given by

$$
\begin{aligned}
& \hat{\sigma}_{\tau}=\frac{1}{2 \pi f} \sqrt{\log \left(2 /\left(1+\sqrt{1-16 \pi^{2} f^{2} \tilde{\sigma}_{\tau}^{2}}\right)\right)} \\
& \hat{\sigma}_{\epsilon}=\sqrt{\tilde{\sigma}_{\epsilon}^{2}-\beta^{2}\left(1+e^{\left.-8 \pi^{2} f^{2} \hat{\sigma}_{\tau}^{2}-2 e^{-4 \pi^{2} f^{2} \hat{\sigma}_{\tau}^{2}}\right) / 2}\right.}
\end{aligned}
$$

and were obtained by equating the coefficients of the constant term and $\left(g^{\prime}\left(t_{i}\right)\right)^{2}$ of (2) and (4).

Table III displays the means of the $100 \hat{\sigma}_{\epsilon}$ and $\hat{\sigma}_{\tau}$ based on the same simulated data used to produce Table II. Table III clearly shows the effectiveness of the adjustments.

Eq. (4) was derived with the assumption that there is no harmonic distortion in the model. If there is harmonic distortion, the exact relationship between $\operatorname{var}\left(y_{i}\right)$ and $\sigma_{\epsilon}$ and $\sigma_{\tau}$ is much more complicated [for example, see (25) in the Appendix for the second-order harmonic case]. However, if the amplitudes corresponding to the harmonics are small relative to the fundamental amplitude, the adjusted estimates of (5) and (6) can still be applied to reduce the bias. To illustrate, we added a secondorder harmonic term, with the amplitude of the harmonic equal to $5 \%$ of the fundamental amplitude, to the sine-wave model used in the simulation above, and compared the two estimation methods. Table IV displays the means of the 100 estimates of the jitter and additive noises for each of the two approximations. It shows that the adjustments in (5) and (6) are useful, even in the presence of harmonic distortion. We also obtained the LS estimate of $\sigma_{\epsilon}$ and $\sigma_{\tau}$ of the simulated data based on (25). Both estimates were unbiased for the simulation parameters and sample sizes considered.

A final remark on the first-order approximation is in order. The first-order approximation is derived based on the assumption that $\sigma_{\tau}$ is small so the high-order terms of $\sigma_{\tau}$ can be ignored. Even if $\sigma_{\tau}$ is small, the bias of the estimate of $\sigma_{\epsilon}$ can still be large. If an estimate of $\sigma_{\epsilon}$ with a small bias is desired, either the exact/adjusted method or other means of obtaining the additive noise should be used. 
TABLE IV

ADDITIVE NOISE AND JiTTER ESTIMATES WITH HARMONIC DISTORTIONS

\begin{tabular}{c|cc|cc|cc}
\hline \multirow{2}{*}{ sample size } & \multicolumn{2}{|c|}{ nominal } & \multicolumn{2}{c|}{ first-order approx. } & \multicolumn{2}{c}{ bias-adjusted } \\
\cline { 2 - 7 } & $\sigma_{\epsilon}(\mathrm{V})$ & $\sigma_{\tau}(\mathrm{ps})$ & $\sigma_{\epsilon}(\mathrm{V})$ & $\sigma_{\tau}(\mathrm{ps})$ & $\sigma_{\epsilon}(\mathrm{V})$ & $\sigma_{\tau}(\mathrm{ps})$ \\
\hline 150 & 0.002 & 1 & 0.0025 & 0.99 & 0.0020 & 1.00 \\
150 & 0.002 & 2 & 0.0061 & 1.94 & 0.0025 & 2.00 \\
300 & 0.002 & 1 & 0.0025 & 0.99 & 0.0021 & 1.00 \\
300 & 0.002 & 2 & 0.0061 & 1.94 & 0.0025 & 2.00 \\
\hline
\end{tabular}

\section{UNCERTAINTY OF TBD ESTIMATES}

Estimation of additive and jitter noises requires the knowledge of the TBD to evaluate the time derivative $g^{\prime}\left(t_{i}\right)$, while the TBD estimation routine needs the estimate of $\sigma_{\epsilon}$ and $\sigma_{\tau}$ to construct the weights for the LS procedure. Thus, an iterative algorithm is used. We begin with a set of equal weights, and then estimate the harmonic distortion, TBD, amplitude, and phase parameters. With these estimates in hand, we estimate the jitter and additive noises and use them to form a new set of weights to obtain the TBD and other parameters. This process is repeated until convergence is attained.

The measurements we use in the iterative procedure consist of a $9.75 \mathrm{GHz}$ signal and nearly quadrature signal, and a 10.25 $\mathrm{GHz}$ signal and nearly quadrature signal. The sinusoidal signals are generated using an inexpensive $100 \mathrm{kHz}-3.2 \mathrm{GHz}$ synthesized signal generator, whose $5 \mathrm{MHz}$ reference is provided by a hydrogen maser that is maintained by the NIST Time and Frequency Division. The synthesized signal is multiplied by a $5 \times$ multiplier and amplified and filtered to ensure spurious harmonics of the input signal are less than $-60 \mathrm{~dB}$ (re: carrier) and spurious harmonics of the output signal are less than $-50 \mathrm{~dB}$ (re: carrier). The oscilloscope is triggered from the fundamental signal generated by the signal generator, and the relative phase of the measured waveform is set by changing the trigger level of the oscilloscope. The model for these 4 waveforms is given by

$$
\begin{aligned}
y_{i j}= & \alpha_{j}+\sum_{\substack{m=1 \\
n_{h}}} \beta_{j m} \\
& \cdot \sin \left(2 \pi m f_{j}\left((i-1) T_{s}+h_{i}+\tau_{i j}\right)+\phi_{j m}\right)+\epsilon_{i j}
\end{aligned}
$$

where $i=1,2, \ldots, n, j=1,2,3,4, n_{h}$ is the harmonic order, $f_{1}=f_{2}=9.75 \mathrm{GHz}, f_{3}=f_{4}=10.25 \mathrm{GHz}, \phi_{21} \approx \phi_{11}+\pi / 2$, and $\phi_{41} \approx \phi_{31}+\pi / 2$. The noises $\epsilon_{i j}$ and $\tau_{i j}$ are assumed to be independent and identically distributed with zero means and standard deviations $\sigma_{\epsilon, j}$ and $\sigma_{\tau, j}$, respectively. The model allows different additive and jitter noise standard deviations for different waveforms.

To improve the TBD estimate we average results from 100 sets of 4 waveforms. Let $\hat{h}_{i k}$ be the individual TBD estimate obtained from the $k$ th set of waveforms, $k=1,2, \ldots, 100$, then the final TBD estimate is given by

$$
\hat{h}_{i}=\frac{1}{100} \sum_{k=1}^{100} \hat{h}_{i k} .
$$

The uncertainty of $\hat{h}_{i}$, expressed in terms of the root mean square error (RMSE) and averaged over the $n$ time points, is

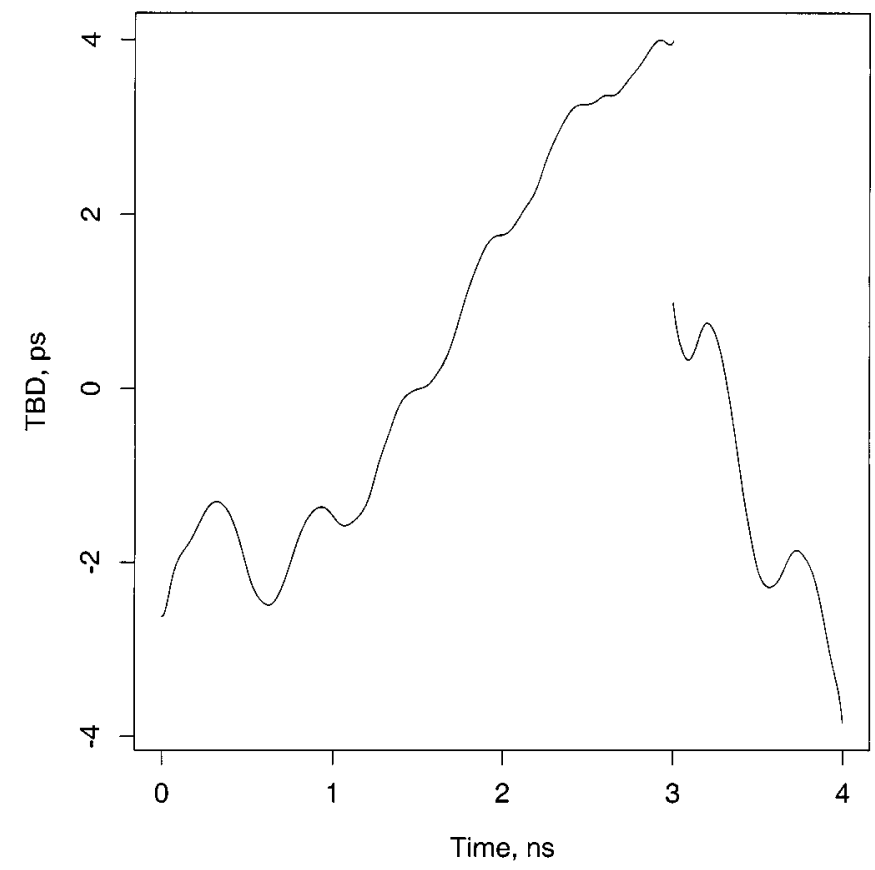

Fig. 1. Timebase distortion used in the simulations.

given by

$$
u(\hat{h})=\sqrt{\frac{1}{n} \sum_{i=1}^{n} \mathrm{E}\left(\hat{h}_{i}-h_{i}\right)^{2}}
$$

where $\mathrm{E}(X)$ is the expected value of $X$. We use simulation experiments to illustrate a method for obtaining the uncertainty of the TBD estimate.

\section{A. Simulation Study 1}

The simulation parameters used here are closely related to those we observe in our laboratory. We use a $4 \mathrm{~ns}$ time window with 4096 points $(n=4096)$. The nominal TBD $h_{i}$, shown in Fig. 1, has a discontinuity at $3 \mathrm{~ns}$ from the starting time. The harmonic order $n_{h}$ used is 3 . We generate 100 sets of four waveforms. Each set contains a $9.75 \mathrm{GHz}$ signal and nearly quadrature signal, and a $10.25 \mathrm{GHz}$ signal and nearly quadrature signal. The fundamental amplitudes for waveforms 1, 2, 3, and 4, are $0.1909 \mathrm{~V}, 0.1911 \mathrm{~V}, 0.2547 \mathrm{~V}$, and $0.2548 \mathrm{~V}$, respectively. The amplitudes corresponding to the second and third harmonics are 10 and $5 \%$ of the fundamental amplitudes. The additive noise standard deviations for waveforms $1,2,3$, and 4 , are 0.00115 $\mathrm{V}(0.60 \%$ of the amplitude), $0.00115 \mathrm{~V}, 0.00067 \mathrm{~V}(0.26 \%)$, and $0.00067 \mathrm{~V}$. The jitter standard deviations for waveforms $1,2,3$, and 4 , are $0.74 \mathrm{ps}$ ( $75.8 \%$ of the sample period), 0.79 ps $(80.9 \%), 0.84$ ps $(86.0 \%)$, and 0.88 ps $(90.1 \%)$. The starting phases of the $j$ th waveform are the same among the 100 sets of data. The iteratively weighted LS procedure described above is used to obtain the TBD estimates $\hat{h}_{i k}$ and then $\hat{h}_{i}$. The process is repeated 200 times. Let $\hat{h}_{i}^{l}$ be the final TBD estimate of the $l$ th simulation sample, $l=1,2, \ldots, 200$, then the mean square 
error of the 200 final TBD estimates, an estimate of $u^{2}(\hat{h})$ in (9), is given by

$$
S^{2}=\frac{1}{4096} \sum_{i=1}^{4096} \frac{1}{200} \sum_{l=1}^{200}\left(\hat{h}_{i}^{l}-h_{i}\right)^{2} .
$$

If we denote $\overline{\hat{h}}_{i}=\sum_{l=1}^{200} \hat{h}_{i}^{l} / 200$, that is, the mean final TBD estimate of the 200 simulation samples, we can further partition $S^{2}$ as

$$
S^{2}=\frac{1}{4096} \sum_{i=1}^{4096}\left(\overline{\hat{h}}_{i}-h_{i}\right)^{2}+\frac{1}{4096} \sum_{i=1}^{4096} \frac{1}{200} \sum_{l=1}^{200}\left(\hat{h}_{i}^{l}-\overline{\hat{h}}_{i}\right)^{2} .
$$

The terms on the right side represent, respectively, the bias and the variance of the final TBD estimate (based on the 200 simulation samples). For the above simulation, we obtain

$$
S^{2}=(0.0431 \mathrm{ps})^{2}=(0.0032 \mathrm{ps})^{2}+(0.0430 \mathrm{ps})^{2} .
$$

The bias is small relative to the variance, and hence the uncertainty of the final TBD estimate can be obtained from the variance of the final TBD estimate. Furthermore, the variance of the final TBD estimate $\hat{h}_{i}$ can be estimated from the variance of the individual TBD estimate $\hat{h}_{i k}, k=1,2, \ldots, 100$. Specifically,

$\widehat{u}^{2}(\hat{h}) \approx \widehat{\operatorname{var}}\left(\hat{h}_{i}\right)=\widehat{\operatorname{var}}\left(\sum_{k=1}^{100} \hat{h}_{i k} / 100\right) \approx \widehat{\operatorname{var}}\left(\hat{h}_{i k}\right) / 100$

where $\widehat{\operatorname{var}}\left(\hat{h}_{i k}\right)$ is the sample variance of the 100 TBD estimates, each of which is obtained using four waveforms. This variance is averaged over the 4096 points and is given by

$$
\widehat{\operatorname{var}}\left(\hat{h}_{i k}\right)=\frac{1}{4096} \sum_{i=1}^{4096} \frac{1}{99} \sum_{k=1}^{100}\left(\hat{h}_{i k}-\hat{h}_{i}\right)^{2} .
$$

Fig. 2 displays the square root of $\widehat{\operatorname{var}}\left(\hat{h}_{i}^{l}\right)$ for $l=$ $1,2, \ldots, 200$. Each of them is an estimate of the RMSE $\hat{\mathrm{u}}(\hat{h})=0.0431 \mathrm{ps}$. The mean of these 200 uncertainty estimates is $0.0428 \mathrm{ps}$. The results indicate that the standard error of the individual TBD estimates is a reasonable estimate of the uncertainty of the final TBD estimate.

\section{B. Simulation Study 2}

Under the model in (7), the uncertainty of the TBD estimate depends on the magnitude of $\sigma_{\epsilon}$ and $\sigma_{\tau}$. If we double the additive noise standard deviations and keep the rest of the simulation parameters the same as used in simulation study 1 , the bias and the variance of the TBD estimate based on the 200 simulation samples are found to be

$$
S^{2}=(0.0453 \mathrm{ps})^{2}=(0.0032 \mathrm{ps})^{2}+(0.0452 \mathrm{ps})^{2} .
$$

The result indicates that the additive noises affect primarily the variance of the TBD estimates.

\section{Simulation Study 3}

If we double the jitter standard deviations and keep the rest of the simulation parameters the same as used in simulation study

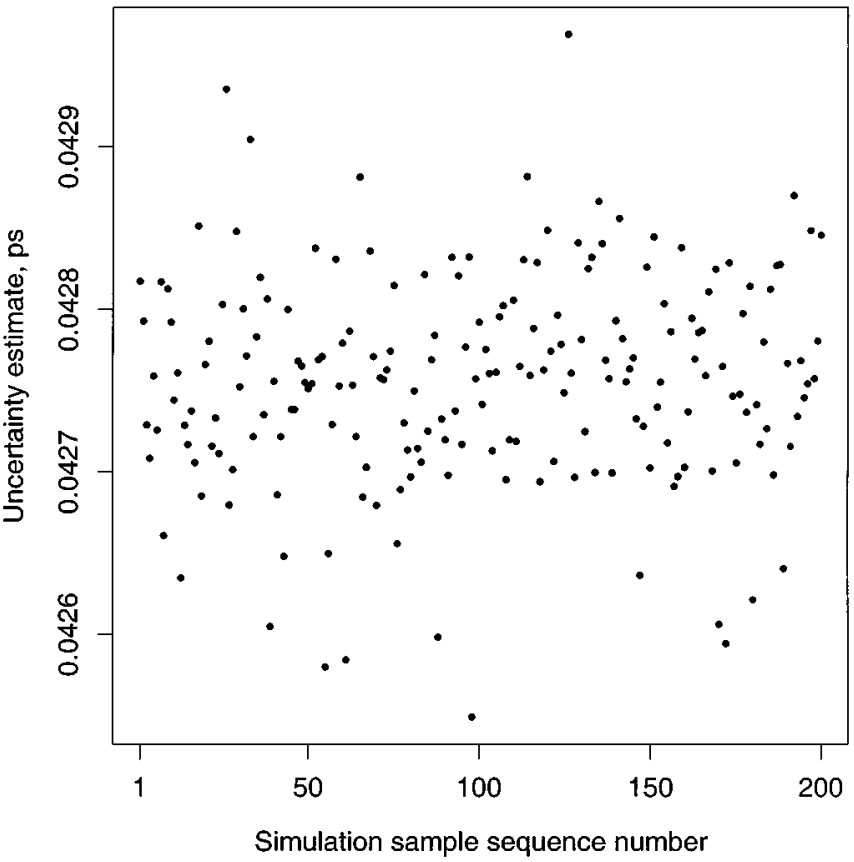

Fig. 2. Uncertainties of the final TBD estimate for the 200 simulation samples.

1, the bias and the variance of the TBD estimate based on the 200 simulation samples are found to be

$$
S^{2}=(0.0857 \mathrm{ps})^{2}=(0.0088 \mathrm{ps})^{2}+(0.0852 \mathrm{ps})^{2} .
$$

Thus, the jitter noises affect both the bias and variance of the TBD estimates, and the effect on the variance is larger compared to the additive noises.

The uncertainty we propose here is integrated over time and ignores any covariance structure in time. We use the uncertainty to monitor the TBD measurements in experiments for estimating the magnitude and phase response of a $50 \mathrm{GHz}$ sampling oscilloscope using the "nose-to-nose" method [8]. Recently, we have observed $\hat{u}(\hat{h})$ in a range of 0.0389 to 0.0392 ps for 13 TBD measurements over a span of 42 days. For future work, we plan to study the effect of TBD uncertainties on the estimation of the magnitude and phase response of sampling oscilloscopes with $50 \mathrm{GHz}$ bandwidth.

\section{SENSITIVITY ANALYSIS OF TBD Estimation}

In general, the starting phase $\phi_{j}$ of the $j$ th waveform in set $k_{1}$ need not be the same as the starting phase $\phi_{j}$ of the $j$ th waveform in set $k_{2}$. However, if the starting phases of the $100 j$ th waveforms are the same, we can then treat these waveforms as repeated measurements and use them to estimate $\sigma_{\epsilon, j}$ and $\sigma_{\tau, j}$. In real measurements, both amplitudes and phases may vary or drift slowly. The estimation results indicate that in our typical $10 \mathrm{~ns}$ measurements, the amplitudes vary within $\pm 0.2 \%$ of their mean values, and the phases drift about $15^{\circ}$, over the 100 sets of waveforms. In this section, we use simulations to study the effects of varying amplitudes and phases, as well as varying jitter noises, on TBD estimation if the waveforms are treated as repeated measurements. 


\section{A. Simulation Study 4}

We employ the same simulation parameters used in simulation study 1 for $h_{i}, f_{j}, n_{h}, \sigma_{\epsilon, j}, \sigma_{\tau, j}$, and elapsed time. We allow the amplitudes to randomly vary within $\pm 1 \%$ of their nominal values, and the phases to drift $31.6^{\circ}$ for waveforms 1 and 2 (corresponding to $90 \mathrm{ps}$ at $9.75 \mathrm{GHz}$ ) and $33.2^{\circ}$ for waveforms 3 and 4 (90 ps at $10.25 \mathrm{GHz}$ ), over the 100 sets of waveforms. The iteratively weighted LS procedure, treating the 100 waveforms as repeated measurements, is used to obtain the TBD estimates. Again, the process is repeated 200 times. The bias and the variance of the TBD estimate based on these simulation samples are found to be

$$
S^{2}=(0.0446 \mathrm{ps})^{2}=(0.0032 \mathrm{ps})^{2}+(0.0445 \mathrm{ps})^{2} .
$$

The estimated RMSE in (17) is 3.5\% larger than that in (12). The mean of the $200 \hat{u}(\hat{h})$ is 0.0483 ps. Thus, the variances of the individual and final TBD estimates are slightly higher than those in simulation study 1 because of varying amplitudes and phases. Overall, the results indicate that a mild violation of the assumption of constant amplitude and phase, used by the TBD estimation procedure, has a small effect on TBD estimation.

\section{B. Simulation Study 5}

In the next simulation experiment, we study the effect of varying jitter errors on TBD estimation. Let $\sigma_{\tau, j k}$ be the jitter standard deviation of waveform $j$ in set $k$, where $j=1,2,3,4$ and $k=1,2, \ldots, 100$. Previously, we assumed that

$$
\sigma_{\tau, j k}=\sigma_{\tau, j} \quad \text { for all } k
$$

If $\sigma_{\tau, j k}$ are different, then the weighting scheme constructed [under the assumption (18)] and used by the estimation procedure is not optimal, resulting in a less accurate TBD estimate. In this simulation, we allow the jitter standard deviations to randomly vary within $\pm 10 \%$ of their nominal values $(0.74,0.79$, $0.84,0.88) \mathrm{ps}$. The iteratively weighted LS procedure, assuming (18), is used to obtain the TBD estimates. The bias and the variance of the TBD estimate based on the 200 simulation samples are found to be

$$
S^{2}=(0.0432 \mathrm{ps})^{2}=(0.0032 \mathrm{ps})^{2}+(0.0430 \mathrm{ps})^{2} .
$$

The estimated RMSE in (19) is almost identical to the RMSE in (12). Thus, a small departure from (18) has no effect on TBD estimation.

If (18) is seriously violated, the correct approach is to estimate additive and jitter variances, and construct the weights separately for each set of four waveforms. Such an approach, however, requires the computation of $\operatorname{var}\left(y_{i}\right)$ in (2). Since repeated measurements are not available in this case, we need to obtain $\operatorname{var}\left(y_{i}\right)$ by other methods, such as repeated measurements from a similar experiment performed previously. Vandeersteen and Pintelon [9] also suggest the use of the square of the residuals from a sine fitting estimation procedure as an estimate of $\operatorname{var}\left(y_{i}\right)$.

\section{CONCLUSION}

We examined the bias of a commonly used method for estimating the additive and jitter noises of sampling oscilloscopes. The bias is not negligible if the sampling frequency is not properly chosen and/or if the jitter noise is not small. We developed a procedure to adjust for the bias. The procedure is based on a model relating the variance of the measured signal and the additive and jitter noises. Simulations were performed to show the effectiveness of the adjustments.

We showed that the bias of a least-squares TBD estimate obtained from multiple sets of waveforms is small relative to the variance of the estimate, allowing us to compute the uncertainty of the TBD estimate from the standard deviation of individual TBD estimates of each set of waveforms. We use this uncertainty to monitor the TBD measurements over time.

\section{APPENDIX}

\section{DERIVATION OF THE VARIANCE IN (4)}

We first state the following preliminaries (for example, see [10]): if $x$ is distributed as a Gaussian with mean $\mu$ and variance $\sigma^{2}$, then

$$
\begin{aligned}
\mathrm{E}(\sin x) & =e^{-\sigma^{2} / 2} \sin \mu \\
\mathrm{E}(\cos x) & =e^{-\sigma^{2} / 2} \cos \mu \\
\operatorname{var}(\sin x) & =\left(1-e^{-2 \sigma^{2}}\right) / 2+e^{-\sigma^{2}}\left(e^{-\sigma^{2}}-1\right) \sin ^{2} \mu \\
\operatorname{var}(\cos x) & =\left(1-e^{-2 \sigma^{2}}\right) / 2+e^{-\sigma^{2}}\left(e^{-\sigma^{2}}-1\right) \cos ^{2} \mu
\end{aligned}
$$

Now

$$
\begin{aligned}
y_{i}= & \alpha+\beta \sin \left(2 \pi f\left(t_{i}+\tau_{i}\right)+\phi\right)+\epsilon_{i} \\
= & \alpha+\beta \sin \left(\omega t_{i}+\phi\right) \cos \left(\omega \tau_{i}\right) \\
& +\beta \cos \left(\omega t_{i}+\phi\right) \sin \left(\omega \tau_{i}\right)+\epsilon_{i}
\end{aligned}
$$

where $\omega=2 \pi f$. Thus, the variance of $y_{i}$ involves the variances of $\cos \left(\omega \tau_{i}\right)$ and $\sin \left(\omega \tau_{i}\right)$, the covariance between $\cos \left(\omega \tau_{i}\right)$, and $\sin \left(\omega \tau_{i}\right)$, and $\sigma_{\epsilon}^{2}$ terms. The first two variance terms can be obtained from (22) and (23) with $\mu=0$ and $\sigma=\omega \sigma_{\tau}$. For the covariance term, we have

$$
\begin{aligned}
\operatorname{cov} & {\left[\sin \left(\omega \tau_{i}\right), \cos \left(\omega \tau_{i}\right)\right] } \\
& =\mathrm{E}\left[\sin \left(\omega \tau_{i}\right) \cos \left(\omega \tau_{i}\right)\right]-\mathrm{E}\left[\sin \left(\omega \tau_{i}\right)\right] \mathrm{E}\left[\cos \left(\omega \tau_{i}\right)\right] \\
& =\mathrm{E}\left[\sin \left(2 \omega \tau_{i}\right)\right] / 2-\mathrm{E}\left[\sin \left(\omega \tau_{i}\right)\right] \mathrm{E}\left[\cos \left(\omega \tau_{i}\right)\right] \\
& =0
\end{aligned}
$$

and Eq. (4) can be easily verified.

Next, we derive the variance of $y_{i}$ for a second-order harmonic model

$$
\begin{aligned}
y_{i}=\alpha+\beta_{1} \sin \left(\omega \left(t_{i}+\right.\right. & \left.\left.\tau_{i}\right)+\phi\right) \\
& +\beta_{2} \sin \left(2 \omega\left(t_{i}+\tau_{i}\right)+\phi\right)+\epsilon_{i} .
\end{aligned}
$$


The variance of $y_{i}$ consists of 4 variances and 6 covariances of $\sin$ and cos variables. The variance terms can be evaluated using (22) and (23). Denote $x=\omega \tau_{i}$ and use the results of

$$
\begin{aligned}
& 4 \sin ^{3} x=3 \sin x-\sin 3 x \\
& 4 \cos ^{3} x=\cos 3 x+3 \cos x
\end{aligned}
$$

the covariance terms can be shown to be

$$
\begin{aligned}
\operatorname{cov}[\sin x, \cos x] & =0 \\
\operatorname{cov}[\sin 2 x, \cos 2 x] & =0 \\
\operatorname{cov}[\sin 2 x, \cos x] & =0 \\
\operatorname{cov}[\sin x, \cos 2 x] & =0 \\
\operatorname{cov}[\sin 2 x, \sin x] & =\left(e^{-\sigma^{2} / 2}-e^{-9 \sigma^{2} / 2}\right) / 2 \\
\operatorname{cov}[\cos 2 x, \cos x] & =\left(e^{-\sigma^{2} / 2}+e^{-9 \sigma^{2} / 2}\right) / 2-e^{-10 \sigma^{2} / 2}
\end{aligned}
$$

Using these results, we have

$$
\begin{aligned}
\operatorname{var}\left(y_{i}\right)= & \beta_{1}^{2}\left(1+e^{-2 \omega^{2} \sigma_{\tau}^{2}}-2 e^{-\omega^{2} \sigma_{\tau}^{2}}\right) / 2 \\
& +\beta_{2}^{2}\left(1+e^{-8 \omega^{2} \sigma_{\tau}^{2}}-2 e^{-4 \omega^{2} \sigma_{\tau}^{2}}\right) / 2+\sigma_{\epsilon}^{2} \\
& +\left(g_{1}^{\prime}\left(t_{i}\right)\right)^{2}\left(e^{-\omega^{2} \sigma_{\tau}^{2}}-e^{-2 \omega^{2} \sigma_{\tau}^{2}}\right) / \omega^{2} \\
& +\left(g_{2}^{\prime}\left(t_{i}\right)\right)^{2}\left(e^{-4 \omega^{2} \sigma_{\tau}^{2}}-e^{-8 \omega^{2} \sigma_{\tau}^{2}}\right) / 4 \omega^{2} \\
& +g_{1}^{\prime}\left(t_{i}\right) g_{2}^{\prime}\left(t_{i}\right)\left(e^{-\omega^{2} \sigma_{\tau}^{2} / 2}-e^{-9 \omega^{2} \sigma_{\tau}^{2} / 2}\right) / 2 \omega^{2} \\
& +g_{1}\left(t_{i}\right) g_{2}\left(t_{i}\right)\left(e^{-\omega^{2} \sigma_{\tau}^{2} / 4}-e^{-9 \omega^{2} \sigma_{\tau}^{2} / 4}\right)^{2}
\end{aligned}
$$

where

$$
\begin{aligned}
& g_{1}\left(t_{i}\right)=\beta_{1} \sin \left(\omega\left(t_{i}+\tau_{i}\right)+\phi\right) \\
& g_{2}\left(t_{i}\right)=\beta_{2} \sin \left(2 \omega\left(t_{i}+\tau_{i}\right)+\phi\right) .
\end{aligned}
$$

\section{ACKNOWLEDGMENT}

The authors gratefully acknowledge many useful communications with F. Verbeyst and G. Vandersteen, and comments and suggestions of G. Stenbakken and J. Splett.

\section{REFERENCES}

[1] R. Pintelon and J. Schoukens, "An improved sine wave fitting procedure for characterizing data acquisition channels," IEEE Trans. Instrum. Meas., vol. 45, pp. 588-593, Apr. 1996.

[2] J. Schoukens, R. Pintelon, and G. Vandersteen, "A sinewave fitting procedure for characterizing data acquisition channels in the presence of time base distortion and time jitter," IEEE Trans. Instrum. Meas., vol. 46, pp. 1005-1010, Aug. 1997.

[3] J. Verspecht, "Accurate spectral estimation based on measurements with a distored-timebase digitizer," IEEE Trans. Instrum. Meas., vol. 43, pp. 210-215, Apr. 1994.

[4] G. N. Stenbakken and J. P. Deyst, "Comparison of time base nonlinearity measurement techniques," IEEE Trans. Instrum. Meas., vol. 47, pp. 34-39, Feb. 1998.

[5] G. Vandersteen, Y. Rolain, and J. Schoukens, "System Identification for data acquisition characterization," in Proc. Instrum. Meas. Technol. Conf. IMTC'98, 1998.
[6] G. N. Stenbakken and J. P. Deyst, "Time-base nonlinearity determination using iterated sine-fit analysis," IEEE Trans. Instrum. Meas., vol. 47, pp. 1056-1061, Oct. 1998

[7] C. M. Wang, P. D. Hale, and K. J. Coakley, "Least-squares estimation of time-base distortion of sampling oscilloscopes," IEEE Trans. Instrum. Meas., vol. 48, pp. 1324-1332, Dec. 1999.

[8] P. D. Hale, T. S. Clement, K. J. Coakley, C. M. Wang, D. C. DeGroot, and A. P. Verdoni, "Estimating the magnitude and phase response of a $50 \mathrm{GHz}$ sampling oscilloscope using the "nose-to-nose' method," in 55th ARFTG Conf. Dig., June 2000.

[9] G. Vandersteen and R. Pintelon, "Maximum likelihood estimator for jitter noise models," in Proc. Instrum. Meas. Techn. Conf. IMTC'99, vol. 2, 1999, pp. 877-879.

[10] J. W. Chapman, "Moments, variances, and covariances of sines and cosines of arguments which are subject to random error," Technometrics, vol. 12, pp. 693-694, 1970

C. M. Wang received the Ph.D. degree in statistics from Colorado State University, Fort Collins, in 1978.

He is a Mathematical Statistician with the Statistical Engineering Division of the National Institute of Standards and Technology (NIST), Boulder, CO. His research interests include interval estimation on variance components, statistical graphics and computing, and the application of statistical methods to physical sciences.

Dr. Wang is a Fellow of the American Statistical Association.

Paul D. Hale received the B.S. in engineering physics in 1985 and the Ph.D. degree in applied physics from the Colorado School of Mines, Golden, in 1989.

He has been with the Optoelectronics Division of National Institute of Standards and Technology (NIST), Boulder, CO, since 1989. He has conducted research in birefringent devices, mode-locked fiber lasers, fiber chromatic dispersion, broadband lasers, interferometry, polarization standards, and high-speed optoelectronic measurements. He is presently Leader of the High-Speed Measurements Project in the Sources and Detectors Group. His present interests are in the area of high-speed optoelectronic and microwave measurements and their calibration.

Dr. Hale, along with a team of four scientists, received the Department of Commerce Gold Medal in 1994 for measuring the cladding diameter optical fibers with an uncertainty of $30 \mathrm{~nm}$. In 1998, he received a Department of Commerce Bronze Medal, along with four other scientists, for developing measurement techniques and standards to determine optical polarization parameters. $\mathrm{He}$ is currently an Associate Editor of the Journal of Lightwave Technology.

Kevin Coakley received the Ph.D. degree in statistics from Stanford University, Stanford, CA, in 1989.

He is a Mathematical Statistician at National Institute of Standards and Technology (NIST), Boulder, CO. His research interests include statistical signal processing, computer intensive statistical methods, and planning and analysis of experiments in physical science and engineering.

Tracy S. Clement received the Ph.D. degree in electrical engineering from Rice University, Houston, TX in 1993. Her Ph.D. research involved developing and studying short wavelength (XUV and VUV) lasers and ultrashort pulse laser systems.

She has been with the Optoelectronics Division of National Institute of Standards and Technology (NIST), Boulder, CO, since 1998. Prior to joining the Optoelectronics Division, she was with JILA, the Quantum Physics Division of NIST, and an Assistant Professor Adjoint in the Department of Physics at the University of Colorado, Boulder. From 1993 to 1995, she was a Director's Postdoctoral Fellow at Los Alamos National Laboratory, Los Alamos, NM. Her current research interests include measuring response of high-speed electro-optic components and ultrashort pulse laser measurements. 